\title{
COVID-19 Pandemic and Electroconvulsive Therapy
}

\author{
Abhishek Singh ${ }^{1} \quad$ Puneet Khanna ${ }^{1}$ \\ ${ }^{1}$ Department of Anesthesiology, Pain Medicine and Critical Care, \\ All India Institute of Medical Sciences, New Delhi, India
}

J Neuroanaesthesiol Crit Care:2020;7:150-153

\section{Introduction}

COVID-19 has been officially declared a pandemic by the World Health Organization. ${ }^{1}$ In this challenging situation, the need to deliver quality care for patients afflicted with serious mental illness is even greater than before. However, the majority of electroconvulsive therapy (ECT) services have been stopped. The reason for this is mainly fear of virus transmission, unavailability of anesthetists, and shortage of drugs and personal protective equipment (PPE). Another important reason cited for closing down ECT is that it is considered as an elective intervention. Providing ECT is important as it not only results in better remission rate, but it is also life-saving for patients not taking any food or are suffering from depression, catatonia, and suicidality. ${ }^{2}$ As rightly said by Espinoza et al, in this COVID era, "ECT is a lifesaving gem." ${ }^{3}$ While most of our energy, healthcare resources, and infrastructure is focused on containing the coronavirus pandemic, there is a need to extend care to those who are afflicted with a serious mental illness and require ECT. But the coronavirus pandemic has placed us at a sustained demand for healthcare infrastructure and essential community resources. Such demands lead to the development of a situation where we need to judiciously allocate our resources, equipment, and manpower for their effective utilization and eventual better outcomes. As a result, ECT, which is considered as an elective procedure, may get suspended, creating a lot of worries for clinicians as well as patients, because ECT is life-saving for some patients, while for others it is essential due to a lack of other available options. Hence, during this time of the pandemic, it is the responsibility of the anesthesiologist to develop a framework and guidelines, according to local needs and infrastructure, to perform ECT, which ensures utmost safety for patients as well as healthcare professionals.

\section{Increased Need for ECT}

ECT is considered as one of the effective treatments for a wide range of psychiatric and neurological illnesses., The recent COVID-19 pandemic is likely to cause a detrimental

Dol https://doi.org/ $10.1055 / \mathrm{s}-0040-1714916$ ISSN 2348-0548.

\author{
Address for correspondence Puneet Khanna, MD, Department of \\ Anesthesiology, Pain Medicine and Critical Care, All India Institute of \\ Medical Sciences, New Delhi 110029, India \\ (e-mail: k.punit@yahoo.com).
}

effect on the mental health of many healthcare professionals and the general public, apart from those who are already under treatment. Mental illness may manifest as a rise in suicide rates in healthcare professionals, survivors of the disease, and persons kept in isolation or quarantine. Hence, we should prepare ourselves for a significant rise in the demand for ECT during and after the COVID-19 pandemic. Data from the SARS-CoV-1 pandemic in 2003 shows that the outbreak significantly affected the mental health of healthcare professionals and survivors. It was noted that 40 to $66 \%$ of survivors suffered from a psychiatric illness, mainly depression and posttraumatic stress disorder (PTSD). They remained symptomatic for as long as 30 months after recovering from the disease..$^{6-8}$ Even those healthcare personnel who were not infected by the SARS virus experienced a varying degree of psychological impairment even months and years after the outbreak. ${ }^{9}$ Jianbo Lai et al surveyed mental health outcomes in healthcare professionals in 34 hospitals across China, who were involved in the care of suspected and confirmed COVID-19 patients. They found that a substantial proportion of healthcare professionals had symptoms of insomnia, anxiety, distress, and depression..$^{10}$

It is our responsibility to judge carefully the risk and benefits associated with ECT during the COVID-19 pandemic. It should be considered as an urgent or semi-urgent procedure for patients afflicted with suicidal tendencies, mania, catatonia, and severe form of depression. ECT is the only treatment option for such patients and it should not be delayed or denied. ${ }^{5,11}$ However, the psychiatrist taking care of these patients must ensure that all possible conservative treatment options have been tried and exhausted before administering ECT as the last resort. 5

\section{Challenges for ECT during COVID-19 Outbreak}

The COVID-19 outbreak has created many significant challenges in performing ECT throughout the world. They range from a patient profile, ECT room issues, and finally infection prevention. 
ECT requires general anesthesia with complete muscle paralysis, which places the anesthesiologist in close contact with the patient's oral and airway secretions. Anaesthesiologist's contact with the patient may last for a few minutes in a closed ECT room and often multiple times over weeks to months. Patients come for ECT to the tertiary care centers from different parts of the country, and they are kept in close contact with other patients in a small room for hours before and after ECT, which poses a significant threat of disease transmission among these patients. Generally, the patients undergoing ECT are older, ${ }^{12}$ have several comorbidities such as hypertension, diabetes, etc. ${ }^{13}$ which increases the risk of mortality if they get infected with COVID-19 during treatment. Additionally, these patients have poor oral and body hygiene due to their mental illness,,$^{14}$ live in crowded places, ${ }^{15}$ and are not able to follow the recommendation of personal hygiene and social distancing to prevent the spread of COVID-19 infection. Other challenges associated with ECT services include frequent rotation of psychiatrist, anesthetist, nurse and other support staff posted for $\mathrm{ECT}$, resulting in an increased risk of ECT staff getting infected by high-risk patients as well as these infected staff, spreading the infection to members of the hospital when they are rotated for duty in other wards or ICU. Another logistic challenge that we may face during a pandemic is a shortage of PPE such as face shield, N95 mask, goggles, gowns, hand sanitizers, and disinfectants. Finally, the major challenge involves having adequate staff to run the service when regular staff get infected or are sent for quarantine.

\section{Patient Selection and ECT Room Preparation during COVID-19 Outbreak}

\section{Testing for COVID-19}

Proper patient selection is very important during a pandemic of such magnitude, as resources are limited and there is a high chance of getting infected during the treatment. It is suggested that those patients who are asymptomatic for COVID-19 disease should be considered for therapy. A nasopharyngeal swab for RT-PCR testing should be performed a day before the ECT for all outpatients. All the admitted patients should be monitored for symptoms of COVID-19 such as fever, cough, or dyspnea, and RT-PCR should be done once in a week. ${ }^{16}$ All COVID-19 suspect patients should undergo RT-PCR testing. All those patients who test positive for the disease should not be allowed to undergo ECT.

\section{ECT Room}

ECT theater should be adequately ventilated with at least 12 air exchanges per hour. ${ }^{17}$ All surfaces and devices within the room should be cleaned with $1.0 \%$ hypochlorite solution after each treatment procedure. The number of healthcare personnel should be kept to an absolute minimum in the ECT room. The team may consist of one anesthetist, one assistant, one psychiatrist, and one nursing staff. Anesthesia work station, monitoring equipment, and ECT machine should be covered with transparent plastic sheets, so that they can be cleaned easily after procedure.

\section{Donning and Doffing PPE}

Separate areas should be marked for donning and doffing of PPE. It is the responsibility of healthcare personnel to pay attention to the sequence of donning and doffing of the PPE. It should be monitored by a trained observer, as errors in the removal of PPE are common, and they are associated with increased risk of transmission of infection among healthcare workers. ${ }^{18,19}$

\section{General Precautions}

- ECT should ideally be performed in a negative pressure isolation room with all the staff wearing full PPE, according to institutional guidelines.

- Gown, face shield, and mask should be changed if they become contaminated with the patient's secretions.

- Proper disinfection of the ECT theater should be done to minimize the chances of cross-infection, with a gap of at least 30 minutes between two patients. ${ }^{20,21}$

- After the procedure, all the equipment should be thoroughly cleaned with $70 \%$ ethanol wipes, and floor should be cleaned with $1.0 \%$ hypochlorite solution.

\section{Recommendations for the Conduct of Safe Anesthesia during COVID-19 Outbreak}

\section{Airway Management}

Airway management for ECT during the COVID-19 outbreak requires efficient coordination of skill and knowledge. The most common procedure of bag-mask ventilation without any airway aid poses a significant risk of viral transmission, especially to the anesthesiologist who is standing at the head end during ECT. Therefore, we recommend ensuring airtight mask seal with high-efficiency particulate air (HEPA) filters attached to the anesthesia circuit, in order to reduce the aerosolization and risk of transmission to healthcare personnel. Since the duration of the procedure is short, we recommend that the senior-most anesthetist should be at the head-end for effective airway management. Supraglottic airway devices and endotracheal tubes are associated with higher chances of coughing and aerosolization. Therefore, their use should be restricted to patients with an anticipated difficult airway.

The barrier method may prove beneficial in airway management during ECT. ${ }^{22,23}$ To date, several devices have been designed for airway management to protect the anesthesiologist from aerosol and droplet contamination during intubation and extubation. ${ }^{24-26}$ These are either intubation boxes made of clear acrylic or plastic sheets covering the head-end of the patients. But most of these devices have not been evaluated by human clinical studies. These devices too come with certain limitations such as inadequate view of the patient's airway, difficulty in positioning the patients for preoxygenation, intubation, extubation, as well as difficulty in removing them if the need arises like loss of airway, vomiting, etc. Therefore, their role in ECT needs further investigation and clinical trials. 


\section{Preoxygenation}

The patient should be adequately preoxygenated before induction of anesthesia to reduce the duration of bag-mask ventilation during apnea. We suggest the following measures for effective preoxygenation:

- Patients should be explained about tight mask fit, so that they do not feel uncomfortable or suffocated.

- Suction with Yankauer tip should be ready.

- Glycopyrrolate (0.2-0.4 mg) may be given to reduce hypersalivation. ${ }^{21,27}$

- The patient may be kept at a slightly reversed Trendelenburg position to improve the functional residual capacity.

- The face mask should be applied while maintaining a good seal.

- If the artificial manual breathing unit (AMBU) is used for ventilation, then it must be ensured that it has attached HEPA filter, which should be changed after each patient.

- The patient is asked to take tidal volume breath for 3 minutes or 8 vital capacity breaths in 60 seconds while maintaining the good mask seal.

\section{Induction and Maintenance of Anesthesia during ECT}

- Anesthesia inducing drug may be given by the nurse or assistant while anesthetist manages the airway.

- Ketamine, methohexital, and etomidate should be used to get the best quality of seizure. ${ }^{21,28}$

- The assistant should be asked to apply plastic sheets at the head-end of the patients just before starting the bag-mask ventilation.

- Gentle bag-mask ventilation with low-tidal volume should be started after complete muscle paralysis

- Ventilatory aid like oropharyngeal airway can be used if there is difficulty in maintaining the patient airway. They should be placed at the end of exhalation under the cover of plastic sheets to prevent the spread of aerosol.

- Just before starting stimulation, ensure complete exhalation before removing the mask and placing the bite block.

- Single-use bite block should be used for each patient.

- Everyone in the ECT room should be clear from the patient when the psychiatrist is delivering the shock.

- Accumulated secretion should be cleared from the oropharynx, but open airway suctioning should be avoided as far as possible.

- Gentle bag-mask ventilation should be restarted with high-quality seal and maintained till the patient can breathe spontaneously.

- To reduce the coughing after coming out of anesthesia, remifentanil can be given during the procedure and IV lignocaine (1-1.5 $\mathrm{mg} / \mathrm{kg}$ of ideal body weight) can be given once the seizure has subsided. ${ }^{20,21,29}$

- Post-ECT, the patient should be given oxygen either by nasal cannula or facemask, depending on institutional protocol.

- Barrier sheets can be removed once the patient is hemodynamically stable, arousable, and not coughing.
- All the patients after undergoing ECT should be allowed to recover in a separate isolation room. The mouth should be covered with a surgical face mask and 6 feet distance maintained between patients. ${ }^{11,21}$

\section{Conclusion}

In this difficult time, we must prepare ourselves to fight against the coronavirus, but at the same time, it is our responsibility to treat the most vulnerable patients. With limited infrastructure, resources, and risk of infection during the pandemic, we should consider ECT as the essential medical procedure and make it available to all the patients already suffering from serious mental illness. The recommendation and suggestions described here can guide anesthesiologists for safely conducting ECT during this viral pandemic.

\section{Conflict of Interest}

None declared.

\section{References}

1 WHO. WHO Director-General's opening remarks at the media briefing on COVID-19 - 11 March 2020. Available at: https:// www.who.int/dg/speeches/detail/who-director-general-sopening-remarks-at-the-media-briefing-on-covid-19-11march-2020. Accessed March 14, 2020

2 Kellner CH, ObbelsJ, Sienaert P. When to consider electroconvulsive therapy (ECT) Acta Psychiatr Scand 2020;141(4):304-315

3 Espinoza RT, Kellner CH, McCall WV. ECT: an essential medical procedure. J ECT 2020. Doi: 10.1097/YCT.0000000000000689

4 Sanghani SN, Petrides G, Kellner CH. Electroconvulsive therapy (ECT) in schizophrenia: a review of recent literature. Curr Opin Psychiatry 2018;31(3):213-222

5 Jelovac A, Kolshus E, McLoughlin DM. Relapse following successful electroconvulsive therapy for major depression: a meta-analysis. Neuropsychopharmacology 2013;38(12): 2467-2474

6 Tapia Granados JA, Diez Roux AV. Life and death during the Great Depression. Proc Natl Acad Sci U S A 2009;106(41): 17290-17295

7 Lee AM, Wong JG, McAlonan GM, et al. Stress and psychological distress among SARS survivors 1 year after the outbreak. Can J Psychiatry 2007;52(4):233-240

8 Kwek SK, Chew WM, Ong KC, et al. Quality of life ansd psychological status in survivors of severe acute respiratory syndrome at 3 months postdischarge. J Psychosom Res 2006;60(5):513-519

9 Maunder RG, Lancee WJ, Balderson KE, et al. Long-term psychological and occupational effects of providing hospital healthcare during SARS outbreak. Emerg Infect Dis 2006;12(12):1924-1932

10 Lai J, Ma S, Wang Y, et al. Factors associated with mental health outcomes among health care workers exposed to Coronavirus disease 2019. JAMA Netw Open 2020;3(3):e203976

11 Tor PC, Phu AHH, Koh DSH, Mok YM. Electroconvulsive therapy in a time of coronavirus disease. J ECT 2020;36(2):80-85

12 Kramer BA. Use of ECT in California, revisited: 1984-1994. J ECT 1999;15(4):245-251

13 CaseyDE. Metabolicissuesand cardiovasculardiseasein patients with psychiatric disorders. Am J Med 2005;118(Suppl 2): 15S-22S

14 Brewer WJ, Edwards J, Anderson V, Robinson T, Pantelis C. Neuropsychological, olfactory, and hygiene deficits in men 
with negative symptom schizophrenia. Biol Psychiatry 1996; 40(10):1021-1031

15 Nelson G, Hall GB, Squire D, Walsh-Bowers RT. Social network transactions of psychiatric patients. Soc Sci Med 1992; 34(4):433-445

16 Sienaert P, Lambrichts S, Popleu L, Van Gerven E, Buggenhout S, Bouckaert F, Electroconvulsive therapy during COVID-19times: our patients cannot wait. Am J Geriatr Psychiatr 2020. Doi: $10.1016 \% 2$ Fj.jagp.2020.04.013

17 World Health Organization P. Infection prevention and control during health care when novel coronavirus $(\mathrm{nCoV})$ infection is suspected. Available at: https://www.who.int/publications-detail/infectionprevention- and-control-during-healthcare-when-novel-coronavirus- (ncov)-infection-is-suspected20200125. Accessed April 8, 2020

18 Okamoto K, Rhee Y, Schoeny M, et al; Centers for Disease Control and Prevention Epicenters Program. Impact of doffing errors on healthcare worker self-contamination when caring for patients on contact precautions. Infect Control Hosp Epidemiol 2019;40(5):559-565

19 Tomas ME, Kundrapu S, Thota P, et al. Contamination of health care personnel during removal of personal protective equipment. JAMA Intern Med 2015;175(12):1904-1910

20 Greenland JR, Michelow MD, Wang L, London MJ. COVID-19 infection: implications for perioperative and critical care physicians. Anesthesiology 2020;132(6):1346-1361

21 Flexman AM, Abcejo AS, Avitsian R, et al. Neuroanesthesia practice during the COVID-19 Pandemic: recommendations
From Society for Neuroscience in Anesthesiology and Critical Care (SNACC) J Neurosurg Anesthesiol 2020;32(3):202-209

22 Canelli R, Connor CW, Gonzalez M, Nozari A, Ortega R. Barrier enclosure during endotracheal intubation. N Engl J Med 2020;382(20):1957-1958

23 Brown S, Patrao F, Verma S, Lean A, Flack S, Polaner D. Barrier system for airway management of COVID-19 patients. Anesth Analg 2020. Doi: 10.1213\%2FANE.0000000000004876

24 Matava CT, Yu J, Denning S. Clear plastic drapes may be effective at limiting aerosolization and droplet spray during extubation: implications for COVID-19. Can J Anaesth 2020;67(7):902-904

25 Malik JS, Jenner C, Ward PA. Maximising application of the aerosol box in protecting healthcare workers during the COVID-19 pandemic. Anaesthesia 2020;75(7):974-975

26 Rahmoune FC, Yahia MM, Hajjej R, Pic S, Chatti K. Protective device during airway management in patients with Coronavirus disease 2019 (COVID-19). Anesthesiology 2020. Doi: $10.1097 / A L N .0000000000003369$

27 Christensen STJ, Staalsø JM, Jørgensen A, Weikop P, Olsen NV, Jørgensen MB. Electro convulsive therapy: Modification of its effect on the autonomic nervous system using anti-cholinergic drugs. Psychiatry Res 2019;271:239-246

28 Rozet I, Rozet M, Borisovskaya A. Anesthesia for electroconvulsive therapy: an update. Curr Anesthesiol Rep 2018;8:290-297

29 Wax RS, Christian MD. Practical recommendations for critical care and anesthesiology teams caring for novel coronavirus (2019-nCoV) patients. Can J Anaesth 2020;67(5):568-576 